\title{
Demencia asociada a VIH y su mutable apariencia en neuroimagen (serie de casos clínicos)
}

\section{Variable appearances in neuroimaging of HIV associated dementia}

\section{(clinical case series)}

Ruth V. Espinosa-Martínez ${ }^{1,2^{*}}$, Claudia C. Calzada-Mendoza ${ }^{2}$ y Sara A. Vázquez-López ${ }^{3}$

${ }^{1}$ Departamento de Resonancia Magnética, Hospital General Centro Médico Nacional La Raza; ${ }^{2}$ Sección de Estudios de Posgrado e Investigación, Escuela

Superior de Medicina, Instituto Politécnico Nacional; ${ }^{3}$ Hospital de Infectología, Centro Médico Nacional La Raza. Ciudad de México, México

\section{RESUMEN}

La infección por el virus de la inmunodeficiencia humana (VIH) en el sistema nervioso central se caracteriza por neuroinvasión, neurotropismo y neurovirulencia, que culminan en muerte y autofagia neuronal, que se expresan con déficit cognitivo. El deterioro cognitivo y de la capacidad de movimiento en pacientes que viven con VIH se denomina desórdenes cognitivos asociados a VIH. Su prevalencia actual está entre el 20\% y el 45\%. La demencia asociada a VIH es la forma grave, cuya expresión en neuroimagen tiene varias modalidades. El objetivo del presente trabajo es la presentación de tres casos descritos desde el punto de vista de neuroimagen.

Palabras clave: Desorden neurocognitivo asociado a VIH. Demencia. Resonancia magnética. VIH. Encefalopatía asociada a VIH.

\section{ABSTRACT}

Human immunodeficiency virus infection involves the brain to cause neuroinvasion, neurotropism, and neurovirulence; that culminate in death and neuronal autophagy. Of these complications the neurocognitive decline associated with HIV infection. Neurocognitive decline complex and functional movement restriction is called neurocognitive 
disorders associated with HIV, and the current prevalence is between $20 \%$ and $45 \%$. HIV Dementia is the most serious form of cognitive disorders and neuroimaging findings are poorly understood and sometimes nonspecific, unless expressed as HIV-associated encephalopathy. The aim of the work was a review of the literature and neuroimaging findings.

Key words: HIV-related cognitive decline impairment. Dementia. Magnetic resonance. HIV. HIV-associated encephalopathy.

\section{INTRODUCCIÓN}

A nivel mundial, aproximadamente 36.9 millones de personas viven con el virus de la inmunodeficiencia humana $(\mathrm{VIH})^{1}$. En México, desde 1983 hasta 2019, se han reportado 87,261. En el segundo trimestre del 2019 se han notificado 4,830 casos vivos, mientras que en el 2018 se reportaron 10,787 casos vivos. El grupo etario más afectado es el de los 15 a los 49 años de edad, con una incidencia de 3.8 por cada 100,000 habitantes $^{2}$. La tasa de mortalidad por 100 mil habitantes para el 2018 es de $3.16^{2}$. Desde 1984 hasta 2019 continúan registrados 89,004 pacientes, de los cuales, 69,472 son del sexo masculino y 19,532 del sexo femenino ${ }^{2}$.

La infección por el VIH causa disfunción neurocognitiva ${ }^{2}$. El deterioro cognitivo asociado a VIH tiene una incidencia del 52\% para déficit cognitivo leve y del $44 \%$ para déficit cognitivo moderado, y el 20\% progresa a demencia relacionada a VIH. Esta última tiene un alto impacto en el entorno laboral, familiar, y social ${ }^{3}$. Se calcula que el $43 \%$ de los casos necesitan cuidado de alto nivel y que casi 9.2 millones de los casos podrían prevenirse para el año 2050 si se desarrollan herramientas de detección temprana ${ }^{3}$.
Se denomina desorden neurocognitivo asociado a VIH (DNAH) al deterioro de la función cerebral, de la capacidad de movimiento y a los cambios en el comportamiento y el estado de ánimo de las personas que viven con $\mathrm{VIH}^{4}$. En el manual diagnostico y estadístico de los trastornos mentales (DSM-V), la alteración neurocognitiva asociada al VIH se agrupa en tres categorías de acuerdo al grado de afectación cognitiva y cambios asociados al funcionamiento diario del individuo (Tabla 1$)^{5}$.

La demencia asociada a VIH es la manifestación más grave de los DNAH debido a la dependencia familiar y social. Antes de la era de la terapia antirretroviral de gran actividad (TARGA), el riesgo acumulado de desarrollar DNAH era de 15 al 20\% ${ }^{6}$.

La prevalencia reportada de los trastornos cognitivos varía del 20 al 45\%, debido a los distintos estadios de la enfermedad. Entre los factores de riesgo se encuentran: evolución prolongada de la infección por VIH, mala adherencia al tratamiento antirretroviral, esquemas de TARGA subóptimos, fracasos terapéuticos reiterados, coinfección por el virus de la hepatitis $C$, cifras bajas de linfocitos T CD4+ (<350 células $/ \mu \mathrm{l})$ y edad > 50 años?. 
Tabla 1. Clasificación y características de los desordenes neurocognitivos asociados a $\mathrm{VIH}^{7}$

\begin{tabular}{|l|l|l|l|}
\hline & $\begin{array}{l}\text { Deterioro } \\
\text { neurocognitivo } \\
\text { asintomático }\end{array}$ & $\begin{array}{l}\text { Desorden } \\
\text { neurocognitivo } \\
\text { leve asociado } \\
\text { al VIH }\end{array}$ & $\begin{array}{l}\text { Demencia } \\
\text { asociada } \\
\text { a VIH }\end{array}$ \\
\hline $\begin{array}{l}\text { Alteración } \\
\text { neurocog- } \\
\text { nitiva }\end{array}$ & $\begin{array}{l}\text { Al menos una } \\
\text { DE en al menos } \\
\text { dos dominios } \\
\text { cognitivos }\end{array}$ & $\begin{array}{l}\text { Al menos una } \\
\text { DE en al menos } \\
\text { dos dominios } \\
\text { cognitivos }\end{array}$ & $\begin{array}{l}\text { Al menos } \\
\text { dos DE en } \\
\text { al menos } \\
\text { dos } \\
\text { dominios } \\
\text { cognitivos }\end{array}$ \\
\hline $\begin{array}{l}\text { Afectación } \\
\text { de las } \\
\text { actividades } \\
\text { de la vida } \\
\text { diaria }\end{array}$ & No & Leves & Importante \\
\hline $\begin{array}{l}\text { Criterios de } \\
\text { delirio/ } \\
\text { demencia }\end{array}$ & No & No & Sí \\
\hline
\end{tabular}

DE: desviación estándar.

\section{FISIOPATOLOGÍA}

La infección por el VIH compromete al cerebro mediante la activación inmunológica crónica en el sistema nervioso central (SNC) o efecto neurotrópico ${ }^{8}$.

El primer paso para la infección del SNC involucra el paso del virus a través de la barrera hematoencefálica (BHE), que se produce por migración transendotelial del VIH dentro de los macrófagos, y se altera la funcionalidad y la permeabilidad de la BHE, lo que aumenta la toxicidad de moléculas provenientes de la sangre ${ }^{9}$. Las moléculas y células involucradas del huésped y del virus desencadenan el daño neurológico por distintos mecanismos. Los más importantes son la disrupción de la homeostasis del calcio, la excitotoxicidad mediada por glutamato, la vía del óxido nítrico y del receptor de N-metil-D-aspartato para finalizar en apoptosis .

\section{NEUROIMAGEN Y DESORDEN NEUROCOGNITIVO ASOCIADO A VIH}

En neuroimagen, hasta un 50\% muestran alteraciones en la resonancia magnética $(\mathrm{RM})^{10}$. Aunque no hay hallazgos patognomónicos en neuroimagen para demencia, inicialmente se requiere un estudio de neuroimagen para excluir enfermedades que puedan imitarla ${ }^{11}$.

Los hallazgos específicos se enumeran a continuación:

- Atrofia de la sustancia gris de la corteza cerebral, en particular la circunvolución cingulada anterior, corteza temporal lateral, área premotora y motora, así como lóbulos parietales y frontales ${ }^{10}$.

- Cambios en la intensidad de la señal y atrofia de la sustancia blanca profunda: algunos pacientes presentan afección focal subcortical de la sustancia blanca que puede asemejarse a las anormalidades observadas en la leucoencefalopatía multifocal progresiva $^{10}$.

- Reducción volumétrica de ganglios basales asociados al deterioro motor, además de los síntomas cognitivos ${ }^{10}$.

- Pérdida de integridad de la sustancia blanca en el cuerpo calloso y la corona radiada ${ }^{10}$.

- En etapas más avanzadas, lesiones confluyentes y simétricas bilaterales en la sustancia blanca, que afectan predominantemente a las regiones periventriculares y a los centros semiovales. Se denomina leucoencefalopatía ${ }^{10}$.

Otra herramienta usada en RM es la espectroscopia de $\mathrm{H}$, técnica que mide los metabolitos cerebrales, y es el método más utilizado para el estudio de a DNAH ${ }^{12}$. Se observan aumento en Cho y Mi en casi todos los casos, 
incluso en etapas tempranas o en individuos totalmente asintomáticos; sin embargo, $\mathrm{N}$ acetilaspartato (NAA) y la relación NAA/ creatina son normales o se alteran según el grado de disfunción cognitiva ${ }^{12}$. La espectroscopía por Resonancia Magnética (MRS) puede ser muy útil en la evaluación, seguimiento y respuesta terapéutica de los pacientes con $\mathrm{DNAH}^{13}$.

El tensor de difusión evalúa la difusión de las moléculas de agua y por ende los tractos de sustancia blanca dañado en pacientes con deterioro cognitivo asociado a VIH. También se pueden evaluar las zonas de activación cerebral en áreas de interés del cerebro y la realización de un paradigma durante la realización del estudio a través de la RM funcional tipo BOLD, en que se observa alta actividad en la región inferior del giro frontal izquierdo y el núcleo caudado en comparación con las personas con VIH negativo ${ }^{14}$.

\section{PRESENTACIÓN DE CASOS CLÍNICOS}

Esta serie consta de tres casos: dos del sexo masculino y uno del sexo femenino. Dos de ellos se manifestaron con leucopatía asociada a VIH, que es la forma crónica, y únicamente un paciente se expresó como leucoencefalopatía multifocal progresiva, que es la forma aguda. Estos casos se exponen a continuación.

\section{Caso 1}

Paciente masculino de 45 años, diestro, con infección por el VIH detectada en el 2000, inicia control y tratamiento en 2015 con zidovudina (AZT), lamivudina (3TC) e indinavir. En agosto de 2018 presentaba CD4 inferior a 160 elementos $/ \mathrm{mm}^{3}$. En septiembre de 2018 comienza con un síndrome confusional, alteraciones conductuales y cefalea moderada, está afebril. Ingresa en el servicio de urgencias por pérdida de fuerza de sus miembros izquierdos y alteraciones del habla, que se agravaron con el curso de los días. El examen mostraba un paciente irritable, con desorientación temporal y espacial, tendencia al mutismo. Signos meníngeos (-). La tomografía computarizada (TC) craneal mostró hipodensidad periatrial simétrica bilateral y atrofia frontotemporal bilateral. La RM de encéfalo contrastado con espectroscopia demostró datos de leucoencefalopatía multifocal progresiva que coexistía con pérdida de volumen cerebral frontotemporal bilateral. El estudio de líquido cefalorraquídeo mostró un citoquímico normal; el estudio por reacción en cadena de la polimerasa (PCR) fue negativo para el grupo herpes (herpes virus 1 , herpes virus 2, citomegalovirus, Epstein Barr) y positivo para el virus JC. La evolución del paciente luego de la comprobación diagnóstica fue tórpida, y se complicó con tuberculosis (TB) pulmonar, que respondió a la terapéutica, y el paciente egresó con limitaciones funcionales y laborales (Figs. 1 y 2).

\section{Caso 2}

Paciente de sexo femenino de 35 años de edad, con 12 años de escolaridad, diestra, separada, con una convivencia estable. En 2010 seropositiva a VIH, renuente a tratamiento. En 2019 ingresa en el servicio de urgencias por delirio paranoide, pero asociado a una gran pasividad (era necesario escogerle la ropa, abandonó las labores domésticas). Se diagnosticó esquizofrenia y recibió risperidona, sin efecto. 


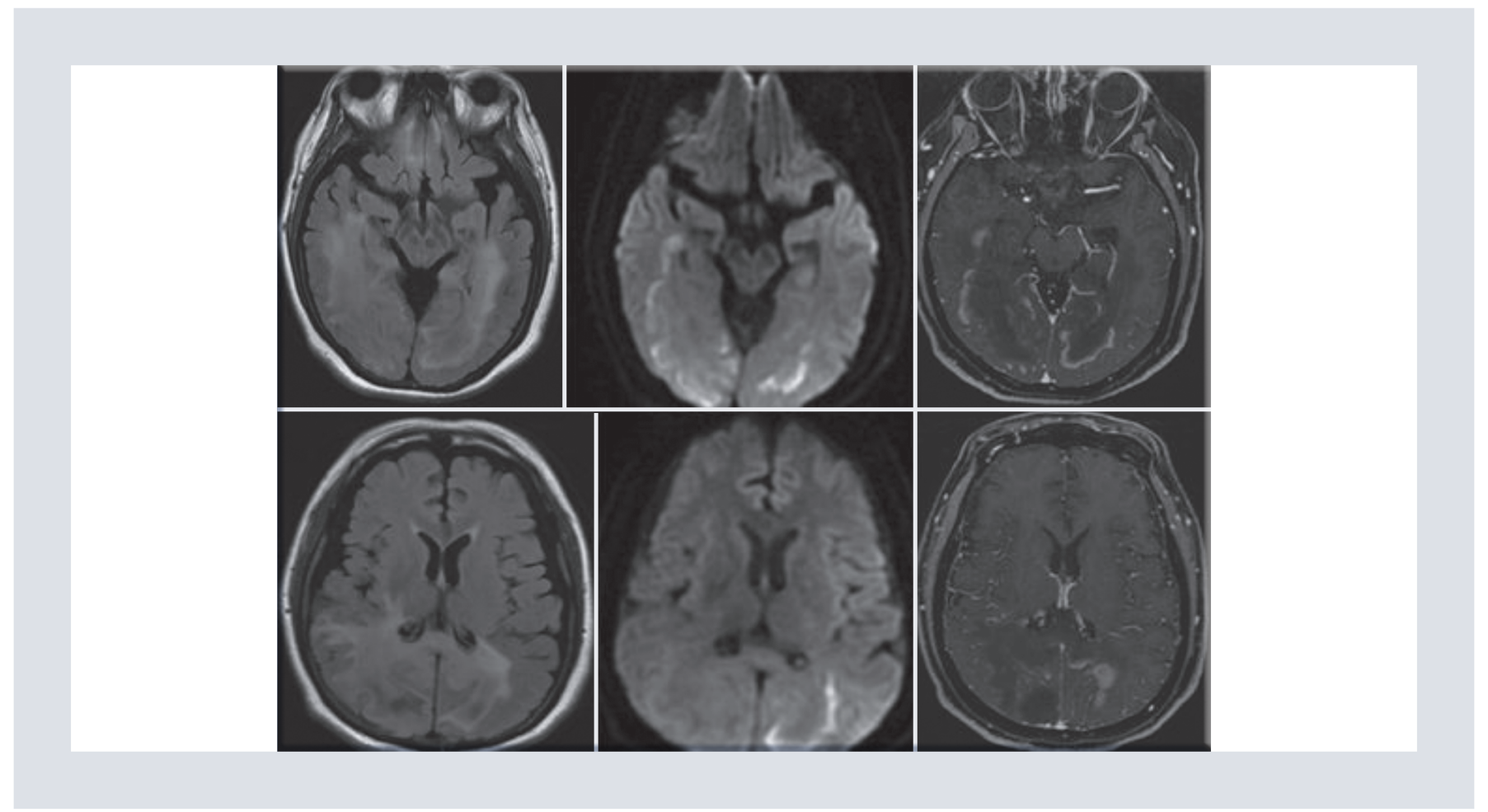

FIGURA 1. Resonancia magnética en secuencias FLAIR, DWI y T13DGd, que en secuencia FLAIR axial sin gadolinio objetiva

hiperintensidad subcortical, periatrial y periventricular, confluente con compromiso del lóbulo parietal y occipital bilateral, asimétrico, que se asocia al efecto de volumen sobre el sistema ventricular, surcos y cisuras, así como cisternas perimesencefálicas, con restricción a la difusión y reforzamiento en su periferia tras la administración de gadolinio. Asimismo, se ven afectadas la corona radiada y los centros semiovales.

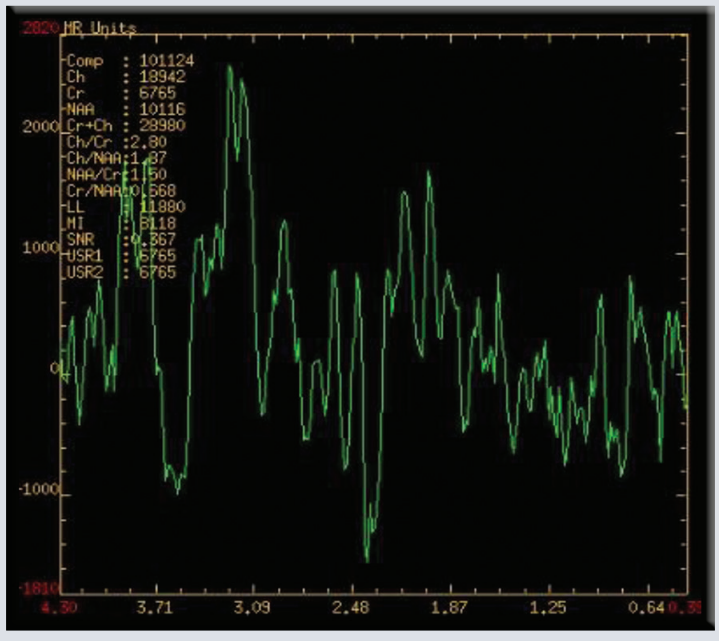

Figura 2. Espectroscopia multivoxel con tiempo de eco de 144 ms, con pico acentuado de colina y mioinositol. Preservación de picos de $\mathrm{N}$-acetilaspartato y creatina. También se ven picos de lípidos.
Un segundo especialista en psiquiatría sospechó un deterioro psicorgánico y la derivó a la clínica de VIH del Hospital de Infectología la Raza. En su evaluación se encontró con hipocinesia sin rigidez ni movimientos anormales; la metría era normal. En la evaluación cognitiva de Montreal (MOCA test) obtuvo solo 10/20 puntos, fracasando en orientación, atención y cálculo, lectoescritura y dibujo, y con respeto relativo de la memoria. Al preguntarle la fecha dijo «no sé»; lo mismo respondió al pedirle restas. Se le realiza una TC que reporta atrofia frontotemporal bilateral. La RM reporta encefalopatía asociada a VIH, atrofia corticosubcortical frontotemporal bilateral, disminución subjetiva de tamaño del hipocampo en forma bilateral (Figs. 3 y 4). 


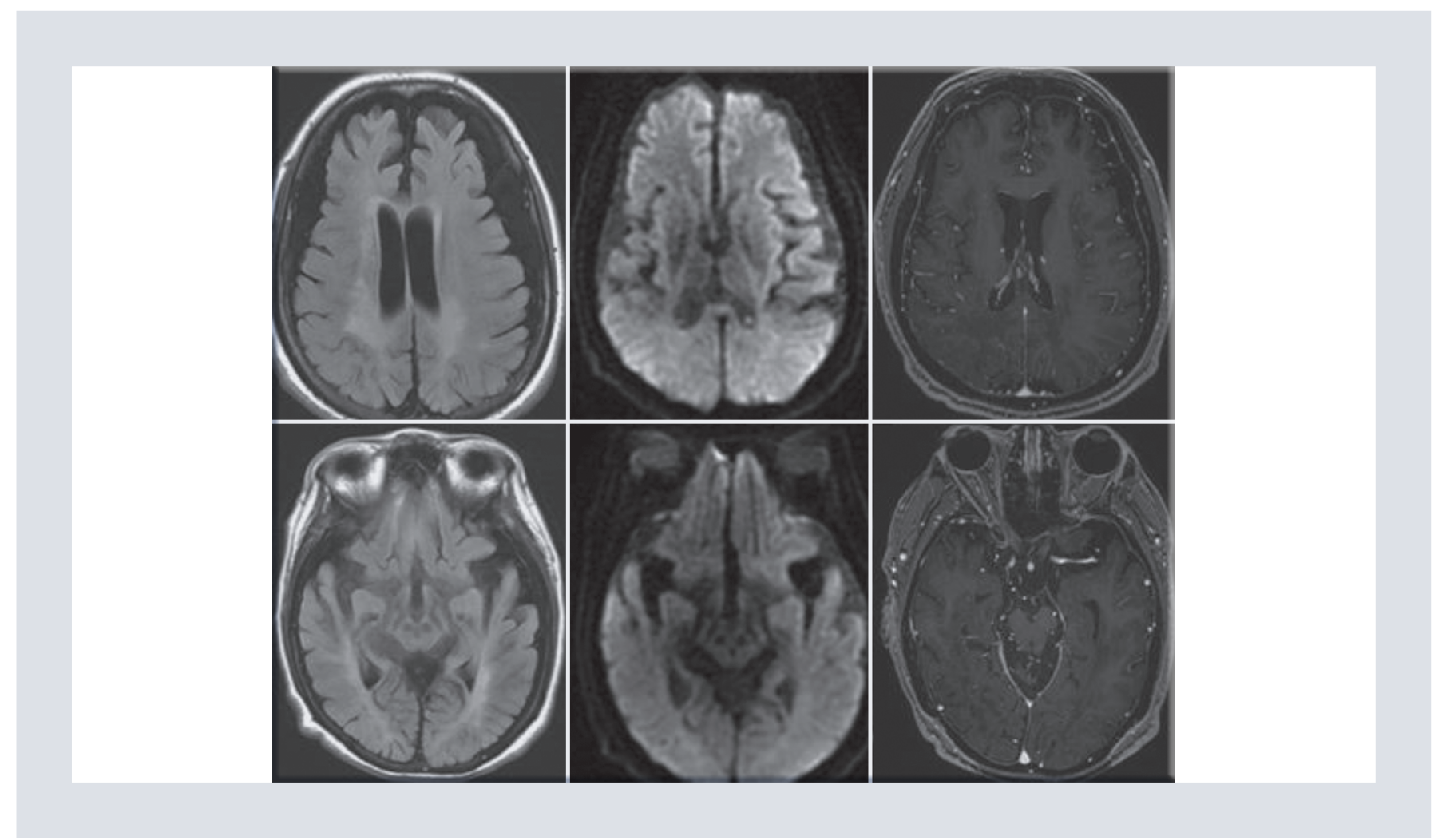

FIGURA 3. Resonancia magnética en secuencias FLAIR, DWI y T13DGd, que en la secuencia FLAIR axial sin gadolinio objetiva hiperintensidad subcortical y periventricular confluente bilateral, asimétrico sin efecto de volumen, sin restricción a la difusión, no hay reforzamiento tras la administración de gadolinio. Marcada disminución de volumen cerebral corticosubcortical frontotemporal bilateral en forma subjetiva. También se identifica marcada atrofia hipocámpica bilateral en forma subjetiva. Sistema ventricular de amplitud conservada.

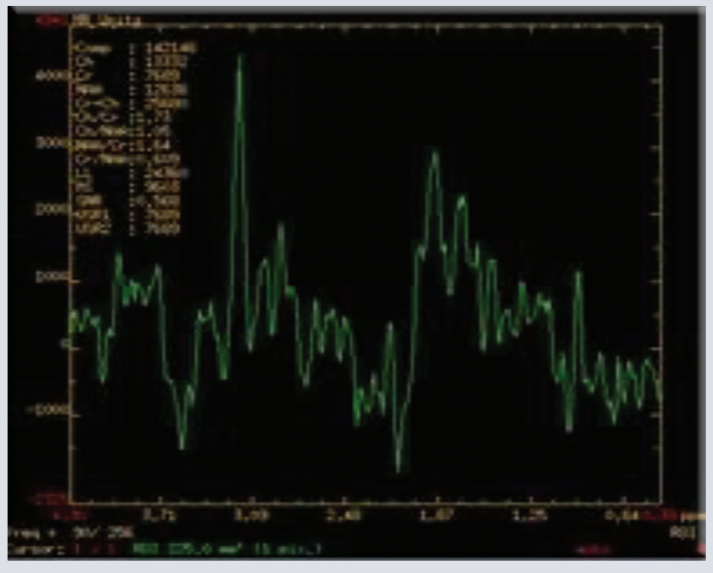

Figura 4. Espectroscopia multivoxel con tiempo de eco de 144 ms, con pico acentuado de colina. Preservación de los picos de $\mathrm{N}$-acetilaspartato y creatina. También se ven picos de lípidos y mioinositol.

\section{Caso 3}

Paciente masculino de 34 años, 14 años de escolaridad, carrera técnica, diestro, soltero, múltiples parejas sexuales. Diagnóstico de infección por VIH desde el 2016, sin TARGA, que ingresó referido de su Hospital General del Instituto Mexicano del Seguro Social (IMSS) por un cuadro de deterioro neurocognitivo progresivo, afasia mixta, amaurosis bilateral y déficit motor en el hemicuerpo derecho. El examen neurológico reveló un déficit motor, cognitivo y del campo visual de evolución progresiva. Se observó, arreflexia osteotendinosa generalizada, sin clonus ni 

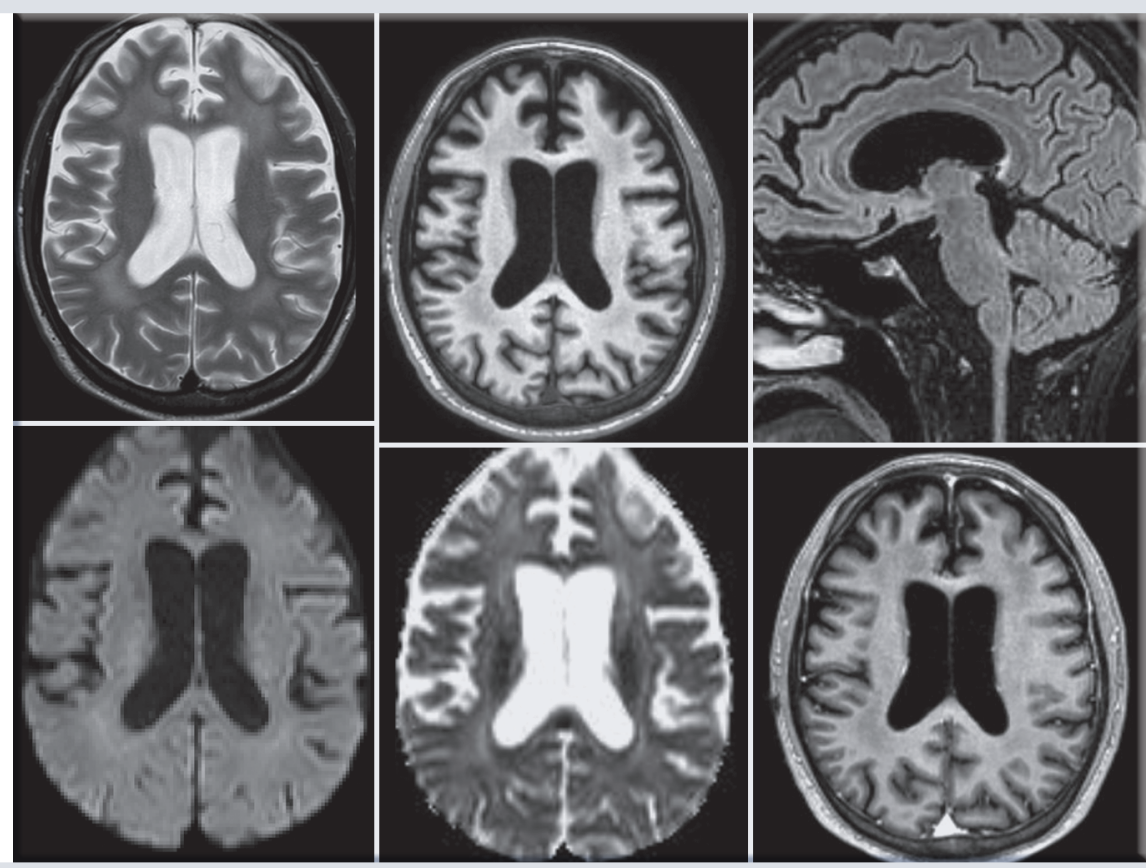

FIgURA 5. Resonancia magnética en secuencias T2, DWI y T13DGd, que en secuencia T2 axial sin gadolinio objetiva hiperintensidad subcortical y periventricular confluente bilateral, asimétrico sin efecto de volumen, sin restricción a la difusión, no hay reforzamiento tras la administración de gadolinio. En la secuencia FLAIR digital se ve marcada atrofia del cuerpo calloso e hiperseñal subcallosa y ventral en el tronco. Disminución de volumen cerebral corticosubcortical frontotemporal bilateral en forma subjetiva. Además de importante atrofia de ambos hipocampos en forma subjetiva. Ventriculomegalia supratentorial compensatoria.

Hoffman y con reflejos plantares indiferentes, pupilas isocóricas, la derecha, hiporreactiva, con amaurosis bilateral, limitación de la mirada conjugada horizontal a la izquierda, con corrección parcial con reflejos oculocefálicos. El recuento de linfocitos T CD4+ fue de 87 cél/ $\mu \mathrm{l}$, con una carga viral plasmática para VIH de 254,000 copias/ml $\left(\log _{10}\right)$. Se efectuó RM de encéfalo contrastada, en la que se observaron datos de encefalopatía asociados al VIH, que se relacionaron a atrofia corticosubcortical frontotemporal, hipotrofia hipocámpica bilateral en forma subjetiva. No hubo realce con el gadolinio (Fig. 5). El examen físico, químico y citológico del líquido cefalorraquídeo evidenció discreta pleocitosis a predominio mononuclear. Los exámenes directos y cultivos para bácilos ácido alcohol-resistentes, hongos y gérmenes comunes fueron negativos, el laboratorio de investigación de enfermedades venereas (VDRL) no reactiva y la reacción en cadena de la polimerasa negativa (PCR) fueron negativas para el virus herpes tipo I y II, citomegalovirus (CMV), virus epstein- Barr (VEB), virus varicela zoster (VZV) y Herpes virus 6 (HHV-6). PCR para JC no reactiva.

\section{DISCUSIÓN}

La demencia asociada a VIH tiene un alto impacto en el entorno laboral, familiar y social, y tiene una incidencia de hasta un $20 \%$ de los casos reportados ${ }^{3}$, esto debido a su 
inespecificidad clínica y neuroimagenológica, así como la disponibilidad y accesibilidad de los métodos de diagnóstico auxiliares como la RM. Con los casos clínicos expuestos se pretende resaltar las diferentes formas de presentación clínica y neurorradiólogica de la demencia asociada a VIH, que si bien la presentación inicial puede ser errática, con la ayuda de la RM cerebral con biomarcadores funcionales se puede hacer una mayor contribución al diagnóstico temprano, y también sirve como un método de exclusión de infecciones oportunistas. En nuestra serie de casos, dos de ellos se presentaron como leucoencefalopatía asociada a VIH, que se traduce en evento crónico, y solo un caso se presentó como un evento agudo dado por leucoencefalopatía multifocal progresiva. En dichos casos, tanto la secuencia contrastada como los biomarcadores funcionales (DWI y espectroscopia), tuvieron un papel primordial para su diagnóstico.

\section{CONCLUSIÓN}

La demencia asociada a VIH es el desorden neurocognitivo de peor pronóstico. Su apariencia en neuroimagen puede ser de diferentes patrones, dependiendo de que la fase evolutiva en la que se encuentre sea aguda o crónica. Además, tiene un gran impacto social, laboral y familiar de los afectados. Pese a su preponderancia y de un panorama incierto hay muy pocas publicaciones radiológicas que analicen sus características en RM usando biomarcadores funcionales.

\section{CONFLICTO DE INTERESES}

Los autores declaran que el análisis de la información y la redacción del presente documento se realizaron bajo total autonomía y sin conflicto de intereses. No se recibió financiamiento para la realización del presente trabajo.

\section{RESPONSABILIDADES ÉTICAS}

Protección de personas y animales. Los autores declaran que para esta investigación no se han realizado experimentos en seres humanos ni en animales.

Confidencialidad de los datos. Los autores declaran que han seguido los protocolos de su centro de trabajo sobre la publicación de datos de pacientes.

\section{Derecho a la privacidad y consentimiento informado. Los autores han obtenido el con- sentimiento informado de los pacientes y/o sujetos referidos en el artículo. Este docu- mento obra en poder del autor de correspon- dencia.}

\section{BIBLIOGRAFÍA}

1. World Health Data Platform. Global Health Observatory: Prevalence of VIH Epidemics. 2019. citado Diciembre 2019]. Disponible en: www.int/gho/ hiv/epidermics-status

2. Censida. Sistema de Vigilancia Epidemiológica de VIH/SIDA. 2019. [citado mayo 2019]. Disponible en www.censida.salud.gob.mx

3. Censida. Dirección General de Epidemiología México. Manual de Procedimientos Estandarizados para la Vigilancia Epidemiológica del VIH-SIDA. 2012. [citado septiembre 2012]. Disponible en www.censida.salud.gob.mx.

4. American Psychiatric Association. Diagnostic and Statistical Manual of Mental Disorders, DSM-V. Washington, DC: APA; 1995.

5. Lamotte Castillo JA. Infección por VIH/sida en el mundo actual. MEDISAN. 2014;18(7):993-1013.

6. Nath A, Schiess N, Venkatesan A, Rumbaugh H, Sacktor N, + Mcarthur J. Evolution of HIV dementia with HIV infection. Int Rev Psych. 2008;20(1):25-31.

7. Rahimian, P, He JJ. HIV/neuroAIDS biomarkers. Progress Neurobiology. 2017;157:117-32.

8. Power C, Hui E, Vivithanaporn P, Acharjee S, Polyak M. Delineating HIVAssociated Neurocognitive Disorders Using Trans- genic Models: The Neuropathogenic Actions of Vpr. J Neuroimmune pharmacol: The Official journal of the Society on Neuroimmune Pharmacology. 2012;7(29):319-31. 
9. Sacktor N, McDermott M, Marder K, Schifitto G, Selnes O, Mcarthur J, et al. HIV-associated cognitive impairment before and after the advent of combination therapy. J Neurovirology. 2002;8:136-42.

10. Haziot M, Barbosa JSP, Vidal JE, de Oliveira F, de Oliveira A. (2015)Neuroimaging of HIV-associated neurocognitive disorders. Dementia \& Neuropsychologia. 2015;9(4):380-4.

11. Petroff O, Duncan J. Magnetic resonance spectroscopy. J Neuroophthalmol. 2008;25:217-226.
12. Ances BM, Hammoud DA. Neuroimaging of HIV-associated neurocognitive disorders (HAND). Curr Opin HIV AIDS. 2014; 9:545-51.

13. Hutchison RM, Womelsdorf T, Allen EA, Bandettini PA, Calhoun VD, Corbetta M, et al. (2013). Dynamic functional connectivity: promise, issues, and interpretations. NeuroImage,. 2013;80:360-78.

14. Elbirt D, Mahlab-Guri K, Bezalel-Rosenberg S, Gill H, Attali M, Asher Illan. HIV-associated neurocognitive disorders (HAND). Isr Med Assoc J. $2015 ; 17: 54-9$ 